1284.24512

N/Sh

Technical Memorandum 86094

\title{
AN X-RAY SURVEY OF VARIABLE RADIO BRIGHT QUASARS
}

M.J. Henriksen, F.E. Marshall, and R.F. Mushotzky

APRIL 1984

National Aeronautics and Space Administration

Goddard Space Flight Center Greenbelt, Maryland 20771 
AN X-RAY SURVEY OF VARIABLE RADIO BRIGHT QUUASARS

\author{
Mark J. Henriksen ${ }^{1}$, Francis E. Marshall, and Richard F. Mushotzky \\ Laboratory for High Energy Astrophysics, NASA/GSFC
}

\begin{abstract}
A sample consisting primarily of radio bright quasars has been observed in X-rays with the Elnsteln Observatory for times ranging from 1500 to 5000 seconds. Detected sources have luminosities ranging from 0.2 to $41.0 \times 10^{45}$ ergs $\mathrm{s}^{-1}$ in the 0.5 to $4.5 \mathrm{kev}$ band. Three of the 14 objects which have been reobserved show flux Increases greater than a factor of 2 on a time scale greater than six months. No varlability was detected during the individual observations. The optical and $x$-ray luminosities are correlated, which suggests a common origin. However, the relationship $\left(L_{x} \sim L_{o p} .89 \pm .15\right)$ found for historic radio variables may be significantly different than that reported for other radio bright sources. Some of the observed $X-r a y$ fluxes are substantially below the predicted self-Compton flux, assuming incoherent synchrotron emission and using VLBI results to constrain the size of the emission region, suggesting relativistic expansion in these sources. Normal CIV emission In two of the sources with an overpredicted Compton component suggests that although they, like BL Lac objects, have highly relativistic material apparently moving at small angle to the line of sight, they have a smaller fraction of the continuum component in the beam.

IAlso Un1versity of Maryland, Astronomy Program
\end{abstract}




\section{INTRODUCTION}

Previous X-ray surveys of quas1-stellar objects (Tananbaum et al. 1979; Ku, Helfand, and Lucy. 1980; Zamoranl et al. 1981) have been made 1n order to determine the radiation mechanisms responsible for the extreme brightness in X-rays as well as in other wavelength regimes. A wide range In the X-ray lumlnosity was found, and a weak correlation was seen between brightness in radio and X-ray. Th1s correlation was tested (Owen et al. 1981) by X-ray observations of a sample of BL Lac objects and quasars showing strong emlssion at mlllimeter wavelengths. As expected, a large number of $X$-ray detections were made and this was interpreted to support the synchrotron self-Compton model. In this model, the photons resulting from the gyration of electrons in a magnetic fleld upscatter off of the same population of electrons and eventually emerge as X-radiation. However, calculations of the Compton luminosity for quasars (Burbidge, Jones, and $0^{-}$Dell 1974) and BL Lac objects (MadejokI and Schwartz 1983; Urry and Mushotzky 1982), showed that the observed X-ray emission was significantly less than expected. In both cases, it was argued that relativistic bulk motion toward the observer resulted in beaming of the radiation and thus an overestimate of the true luminosity of the source.

In designing our survey, we hoped to test further the jet hypothesis

for quasars by observing those bright in radio emission, reasoning that if the primary difference between radio bright and radio weak 18 due to alignment of a jet with the observers line of sight (Scheuer and Readhead 1979), then the over-estimation of the Compton luminosity should be found for a 81gnificant number of these radio bright objects. In addition, if the synchrotron self-Compton mechanlsm were actually at work, then self conslstency of thls model would require that the objects most variable in 
radio would be the most likely to show varlabllity 1n X-rays. Thus, the members of the present sample were chosen from a compliation of bursting radio sources and those showing flux vartations at low frequencles (Marscher et al. 1979). 


\section{OBSERVATIONS}

The Imaging Proportional Counter (IPC) on the Elnsteln Observatory (Glaccont et al. 1979) was used to observe the sample In X-rays. Th1s detector, which has a fleld of view of about one square degree and a spatial resolution of about 1 arcmin, 18 most sensitive in the .5 to 3 kev energy band. Table 1 contalns the count rates observed in this energy band.

The sources observed were chosen primarily because of their historic radio varlability. Observation lengths range from 1500 to 5000 seconds. In addition, 14 objects were reobserved $81 x$ months or one year later. Since the IPC records photon arrival times, we were able to search for variability over a wide range of timescales.

The sample consists of 24 quasars, 2 radio galaxies and 9 unidentified varlable radio sources. The redshift is known for 18 sources and ranges from .059 to 2.286. The redshifts were obtalned from Kuhr et al. (1981). Identification of the radio sources as a quasar or a radio galaxy for objects without measured redshift 18 also from Kuhr et al.

We found that $77 x$ of the objects were detected as X-ray sources. This detection rate 18 comparable to the comblned surveys of Zamorant et al. (1981) and Ku, Helfand, and Lucy (1980). Kembhav1 and Fablan (1981) report that the comblned surveys show 827 of the radio loud quasars were detected as opposed to $22 \%$ of the radio quiet (all others). We use the definition of radio loud given by Zamorani et al. $\alpha_{\text {ro }}>0.35$ where $\left.a_{\text {ro }}=-\log \left[\mathrm{L}_{r} / \mathrm{L}_{0}\right] / \log \left[\nu_{r} / \nu_{0}\right]\right)$. All of the members of the present sample. are radio loud and the detection rate 18 similarly high. The radio luminosities used to calculate the radio to optical spectral index utilize nearly simultaneous (to the X-ray observation) radio fluxes at $8 \mathrm{GHz}$. 
provided by M. Aller of the University of Michigan.

The $\mathrm{X}$-ray luminosity in the 0.5 to $4.5 \mathrm{kev}$ band, at the source, 18 calculated (Table 2) for the objects with known redohtft. The procedure for the luminosity calculation is that given in Tananbaum et al. (1979). We assume a hydrogen column density of $3 \times 10^{20} \mathrm{~cm}^{-2}$, and a power-law spectrum with energy spectral index of 0.5 . Because of the difficulties In getting good spectral fits from the IPC, the hardness ratio (defined as (counts in $1.2-3.0 \mathrm{kev}$ band)/(counts in $0.5-1.2 \mathrm{kev}$ band)) 1s used to Indicate the approximate slope of the power law. The average hardness ratio for detected sources is consistent with a spectral index of 0.4 or 0.5 (Zamorant et al. 1981) but In general for these weak detectlons it 18 not well determined. More importantly though, the distribution of the hardness ratio is consistent, within random counting errors, with that found for other quasars with a wder range in radio luminosity.

We have assumed a $30 \%$ uncertainty in the luminosity, due to uncertalnties in the IPC gain and possible differences in the spectrum from the assumed $\alpha=0.5$, In addition to the statistical error.

For sources not detected, upper 11mits are given. The upper 11mits are 3 sigma above the actual flux at the object's position. The $x$-ray luminosities range from $2 \times 10^{44}$ to $4 \times 10^{46} \mathrm{ergs}^{-1}$.

Optical luminosities are calculated based on magnitudes which are not measured simultaneously with the $X$-ray and radio observations. Th1s will Introduce an uncertainty Into the. optical luminosity for the quasars which are most variable, such as NRAO 530. Based on non-simultaneous opt1cal coverage of a few of our sources (discussion section of this paper), there Is resson to belleve that this uncertainty is significant. A parameter which ls often used to characterize the relative brightness in the optical 
and $x$-radiation 18 the optical to $x$-ray spectral index ( $\alpha_{\text {ox }}=$ $\left.-\log \left[L_{x}(2 \mathrm{kev}) / L_{0}(2500 A)\right] / 2.608\right)$. For the 13 detected quasars in th1s sample with known redshift, the average $\alpha_{o x} 181.29$ with a standard deviation of .14; the undetected objects typically have a larger $\alpha_{o x}$. Comparison to other extragalactic X-ray sources (see F1gure 1) shows that this value of $\alpha_{o x} 18$ similar to that previously found for radio bright quasars (Zamoranl et al. 1981), and nearby radlo bright objects such as $\mathrm{N}$-galaxies and Bl Lacs (Ku, Helfand, and Lucy).

\section{Discussion}

\section{(A) Synchrotron-Self Compton Model}

We assume Incoherent synchrotron self-Compton for our sources and calculate the Compton luminosity (Marscher 1983). The distribution of electrons is assumed to be 1sotroplc and given by a power law with spectral Index 2.0. The Compton flux is not very sensitive to the spectral index. For example, In the case of DA406, an electron spectral Index of 1.5 would decrease the flux roughly by factor of 2 wh1le an electron spectral index of 2.5 would increase the flux by a factor of 2 . In the most 1nteresting cases, such as DA406, 0400+25, OR103, and 0738+31, the uncertainty in the predicted flux is Insigniflcant compared to the disagreement with the observed flux. The flux $\left(\operatorname{ergs~} \mathrm{cm}^{-2} \mathrm{sec}^{-1}\right)$ in the .5 to $4.5 \mathrm{kev}$ band 18 given by equation (A) for photon spectral index 1.5 .

$$
F(.5-4.5 \mathrm{kev})=5.8 \times 10^{-10} \ln \left(\frac{\nu_{2}}{\nu_{m}}\right) s_{m}^{5} \theta_{\nu_{m}}^{-8}{ }^{-6.5}(1+z)^{5} \delta^{-5}
$$

\footnotetext{
$v_{\mathrm{m}}$ is the turnover frequency, assumed due to self-absorption, in $\mathrm{GHz}$
} 
$S_{m}$ is the flux at $v_{m}$, obtained by extrapolation of the transparent part of the spectrum back to the turnover frequency, In Janskys

$\theta$ is the angular diameter of the emitting region in milliarcsec

$\delta$ 1s the Doppler factor necessary to reduce the flux down to observed $11 \mathrm{~m} 1 \mathrm{ts}$

$=[\Gamma(1-\beta \cos \theta)]^{-1}$ where $\beta=v / c, \quad \Gamma=\left(1-\beta^{2}\right)^{-1 / 2}$

To measure $v_{m}$ and $S_{m}$ accurately for variable radio sources, it is preferable to use simultaneous radio fluxes at a number of frequencies, typlcally ranging from 1 to $100 \mathrm{GHz}$; the primary constralnt being that the turnover in the spectrum is visible. We use spectra published by 0wen, Spangler, and Cotton (1980) and Owen et al. (1978), for 7 of 9 objects in - table 3. For two sources we use the spectra in Kuhr et al. (1981). The self-Compton flux is most sensitive to the angular size of the emitting region. We use VLBI fringe visibilities (FIgure 2) and following Marscher (1983), assume a spherical source to calculate an upper limit on the size of the emitting region. The calculated sizes are upper limfts for two reasons. The resolution, given by the inverse of the separation of the Interferometer elements, is on the order of 1 mas. This is also the typical source extent. Radio maps which partially resolve the compact cores of two quasars, NRAO 530 and NRAO 140 (Marøcher and Brodertck 1981), show two compact components which are tenths of a mil11arcsec in diameter, separated by 1-2 mas. It may be that the fringe visibilities used here do not resolve these two components, which could be present in many 
quasars. Also, there may be multiple, discrete components of varylng opt1cal depth with the X-rays originating in an unresolved component (or one that is obscured by more diffuse emission) in close proximity to the "central machine". The spectrum resulting from this "onion-skin" model is usually flat (the opaque section of the spectrum of one layer 18 overlayed by the transparent part of the next outer layer).

No turnover 18 visible in the flat radio spectrum observed (In the sense that the "classic" opaque spectrum 1s not observed) for CTA26, 1739+52, NRAO 530,4C31.63, and DA393. However, the spectra of DA393 and $1739+52$ flatten out at low frequencles. Such spectra can be produced by multiple synchrotron components with different turnover frequencles. For these 2 sources, we choose the frequency at which the spectrum begins to flatten. We have used the largest turnover frequency for which equation $A$ is valld assuming $\nu_{2}$ is $300 \mathrm{GHz}$, for the remalning 3 sources (Marscher 1983). Smaller values of the turnover frequency would require larger Doppler factors. The source DA 406 has a rad10 spectral index of 0.23 . The lowest observed frequency, at which no turnover 18 seen, is used as an upper limit for the turnover frequency. An 1ncorrect cholce of this parameter may account for the extremely large predicted Compton luminosity.

Ennis et. al (1982) found that extrapolation of the radio spectrum predicts the flux at $1 \mathrm{~mm}$ accurately for radio bright quasars. Though the number of sources in his sample is small, if this result extends to all radio bright quasars, then $300 \mathrm{Ghz}$ can be used as a lower 11mit to the break in the synchrotron spectrum $\left(v_{2}\right)$ used in equation (A).

For 5 of the quasars In Table 3, a Compton flux 18 predicted which is 81gniflcantly greater than the observed level. In the case of $1739+52$ the 
observed flux is lower than the predicted flux by a factor of 491, for $0738+31$ the observed flux is lower than that predicted by a factor of $2.6 \times 10^{5}$, for OR103 it is lower by $3.4 \times 10^{3}$, for $0400+25$ it is lower by a factor of $1.9 \times 10^{6}$, and the most severe case, DA406, the Compton flux exceeds observation by $10^{11}$. The predicted flux is a lower 11mit; if the emitting region is smaller, then the descrepancy would be even greater. The predicted flux can be reconclled with the observed flux if the emftting region is approaching us at a highly relativistic velocity (as in a jet at small angle to the IIne of sight). The Doppler factor is given In Table 3 which reduces the predicted X-ray flux to what we observe. Eigure 3 shows the acceptable combinations of jet velocity and viewing angle for these four sources.

The range of $\delta$ inferred for these objects is quite simflar to that seen in BL Lac objects (Madejsk1 and Schwartz, 1983) whtch have $.35<\delta<$ 30 and typically in the range 2 to 6 , while we have $\delta_{a v}=5.8$ (excluding DA406).

Using the VLBI to measure the source size used in this calculation is an Improvement over the size derived from light travel time arguments used to infer an upper limit from the varlability since the VLBI data remove the dependence on the Hubble constant. The predicted Compton flux varies as the elghth power of the size (for $\alpha=0.5$ ), so an uncertalnty of 2 would lead to an error of 256 in the flux. Th1s 1s, of course, not critical for the most extreme cases such as $0400+25$. However, to observe (albelt indirectly) a wide distribution of jet angles would require distance independent sizes. It ls also interesting to note that a wide range, $>10^{12}$, of X-ray fluxes, is predicted by this model in the absence of beaming. 


\section{(B) Jets and Emission Lines}

In BL Lac objects, the presence of Jets and the lack of strong line emfssion suggests a simple connection (Blandford and Rees 1978). Consider a model for the optical emission line region in which a power law continum source lonlzes a surrounding spherlcal distribution of clouds. If the continuum source lo expanding in a relativistic fet, then the continuum energy 18 beamed Into a small angle $(\theta \sim 1 / \delta)$. A sma11 volume (compared to lsotroplc irradiation of the clouds) of the clouds is then photolonized. The emission lines would then be lost in the continum or be very weak, depending on the bulk velocity. However, this cannot account for quasars such as DA406 $(1611+34)$ and $0400+25$ which show evidence for Jets (this paper) and also have CIV equivalent widths (Wampler et al. 1983) similar to other quasars (for their optical luminosity). It may be that only a fraction of the continuum is actually beamed and that the remainder Irradiates the surrounding clouds in a spherically symmetric fashion (Perola 1984). Under this hypothes18, objects such as DA406 and $0400+25$ have only a small fraction of their continum beamed so that most of the power law continum contributes to photolonization of the emfssion line region giving simflar equivalent widths to unbeamed objects. Part of the observed X-ray flux probably comes from the unbeamed component; thus $\delta$ is truly underestimated. Th1s 18 clear from the fact that the rat1o of $X$-ray to optical luminosity 18 normal for DA406 and $0400+25$; one would not call them unusual unless one had VLBI data. The fraction of the power law component which is in the fet then 18 a free parameter which in the case of BL Lacs is large enough to drown the emission lines in the continum or in the case of DA406 it 18 small enough to give normal 11nes. However, self consistency of this scenario requires that the fraction of the the core 
moving relativistically not be too small. The Compton problem for varlable radio bright sources follows from the fact that we are observing a region of small angular extent, presumably a jet: thus the observed radio flux from the jet must dominate the flux from the unbeamed component.

(C) Optical and X-ray Correlation

An earlier survey of radio selected Quasars (Zamorant et al 1981), found that the ratio of X-ray to optical flux varied by a factor of $>300$, but that the fluxes were weakly correlated (correlation coefficlent, $r=$ $.45)$.

We applied a least squares fit to the optical and X-ray monochromatic luminosities, using flux levels at the sources position in the case of non-detections. The distribution and best fit line is given In Figure 4. The correlation coefficient is .92 . However, the reduced ch1-square is 4.2, implying that the linear fit is not a good model.

By adding an uncertainty into the $X$-ray Iuminosity, equal to some percent of the luminosity, a good fit can be achieved. An $80 \%$ systematic error is necessary to give a good fit. This means that the optical luminosity predicts the X-ray luminosity to within $80 \%$. This would be a surprisingly good correlation if the dominant mechanism for the $\mathrm{X}$-rays were Compton emission (without beaming), since the range in physical parameters from source to source would lead to a wide distribution in Compton luminosities. The most obvious explanation is that for radio bright quasars, the alignment of a jet at small angle to the observer reduces the contribution of the Compton process to the $\mathrm{X}-$ ray emission so that it is not the dominant part of the $X$-rays. The 
close correlation between the optical and X-ray luminosities 18 probably due to their having a common production mechanism: synchrotron radiation (Ku, Helfand, and Lucy 1980).

One of the possible explanations for the fact that the systematic uncertalnty is as large as 807 is that these objects vary optically in a component which 18 not correlated with the X-ray emlssion. Malkan and Sargent (1982) have found that the continuum in the UV around 2500A 1s well fitted by a power law plus blackbody and the Balmer continuum. They also find that the non-thermal, power law component accurately predlcts the $2 \mathrm{kev} X$-ray flux. The thermal component is belleved to be highly varlable, though this decreases for more lumfnous quasars (Malkan 1983). Furthermore, the rat1o of blackbody to non-thermal flux is found to Increase with luminosity. So, the thermal flux would be different for objects of different luminosity and may also vary in time.

Optical data, provided by J. Pollock, of the University of Florida, for some of our sources shows a wide range in optical varlability. For example, CTA 26 brightened by a factor of 2.5 in 3 weeks, OT 081 dimmed by 4.5 times in one year, and the optically violent variable NRAO 530 brightened by a factor of 4 within 2 months. S1x sources were reobserved in X-rays after 6 months and 8 after 1 year. A variation of greater than a factor of 2 in the count rate 18 seen in 3 of the 6 objects reobserved in 6 months. None of the 8 objects reobserved after 1 year varled by more than 50\%, and none of the ent1re sample 18 varlable over the length of a single observation, typically 1 hour. Though the opt1cal and X-ray observations are not simultaneous, it appears that the $X$-rays are probably not characterlzed by flux varlations comparable in magnitude to the optical flux varlations over 
simflar timescales. This could be understood if the non-thermal, optical and the X-ray component are highly correlated while the blackbody component is, not correlated to the X-ray and suffers large flux varlations. The radio data do not show flux changes greater than $50 \%$ for those sources with multiple X-ray observations. However, because of the combined systematic errors in the IPC and random errors for sources with few counts, we can not rule out that the radio and Xray are both variable. Zamorani (1983) found that quasars typlcally varied In flux by less than a factor of 2 in six months. Madejeski and Schwartz (1983) found that 11 out of $16 \mathrm{BL}$ Lacs were variable by more than a factor of 2 . There is a weak indication that the quasars reported here may be more similar to $B L$ Lacs in their characteristic Xray varlability.

There are other variables which may reduce the $\mathrm{L}_{x}$ and $\mathrm{L}_{\text {op }}$ correlation such as an $\mathrm{L}_{x}$ dependence on $\mathrm{L}_{x}$ (Owen et al 1980, Tananbaum et a1. 1982), a possible weak dependence of $L_{x} / L_{o p}$ on redshift and a dependence of $\mathrm{L}_{x} / \mathrm{L}_{\text {op }}$ on $\mathrm{L}_{\text {op }}$ (Relchert et al. 1982).

The best fit relationship for our sample is $\mathrm{L}_{\mathrm{X}} \sim \mathrm{L}_{\mathrm{op}} \cdot 89 \pm .15$. Th1s Is 2 sigma different from the correlation $\left(L_{x} \sim L_{o p} \cdot 47 \pm .15\right)$ reported by Tananbaum et al. (1982) for the 3CR sample. We applied the least: squares analysis to the $3 \mathrm{CR}$ sample and found that a good fit, $\mathrm{L}_{\mathrm{X}}$ $L_{\text {op }} .51 \pm .11$, is obtalned for a systematic uncertainty of $100 \%$. This aggrees with the correlation found by Tananbaum et al.. The distribution in $\alpha_{\text {ro }}$ indicates that the $3 C R$ sample is simflar to our sample in radio brightness. The apparent difference with the 3CR sample may be related to the fact that the sample reported here was chosen for historic radio variability. In this respect, our sample is similar to 
that of Owen et al. We applied the same linear regression anslysis to the 12 sources in his sample with known redshift. Very good agreement with our result 18 found $\left(L_{x} \sim L_{o p} \cdot 86 \pm .14\right)$. Th1s suggests that the relationship between $L_{x}$ and $L_{o p}$ is not unlque for radio bright quasars and that variable radio sources may be significantly different than those found in the $3 C R$ sample. An explanation suggested by Tananbaum et al. Is that the $3 \mathrm{CR}$ sources show more of an extended component with. steep spectrum and the variable sources emphasize the nuclear, flat spectrum component (a source of greater X-ray production). Th1s nuclear region 18 probably a mixture of jet and 18otroplc radiation though the lack of short term varlability in the $X$-ray band may Indicate that the $x$-ray emission from the fet 1 s dominated by the 1sotropic radiator. The relationshlp we find then Indicates that for a given optical luminosity, the varlable sources have a higher X-ray luminosity. Th1s 18 qualitatively consistent with the 1dea that the relative 1mportance of the nuclear and extended components is different in the two samples.

\section{SUMMARY}

The apparent relationship between the optical and the $x$-ray luminosities suggests a common origin. From the comparison of the calculated Compton luminosity and the observed levels, we find that the Compton component 18 suppressed in at least some of the strong radio quasars, perhaps due to near alignment of a fet.

If one accepts that a beamed continuum can account for the lack of strong emission Ifnes in BL Lacs, then the presence of normal CIV emission in quasars with highly relativistic fets allgned nearly along the line of sight, suggests that the fraction of the continuum component 
which 18 beamed varles amongst these sources and that BL Lac type objects may be quasars in which such a large fraction of the power law continuum 18 beamed that the emission lines are lost. The other extreme,' In which a small fraction of the concinuum is in the jet, would be quasars such as $1611+34$ and $0400+25$.

We find that the dependence of $X$-ray luminosity on optical luminosity may be significantly different for radio sources which show variability in thefr radio emlssion.

\section{ACKNOWLEDGEMENTS}

MJH would like to thank E. Boldt and T.A. Mathews for making this research possible. We thank D. Schaffer for providing unpublished VLBI observations, J. Pollock for optical data, and M. Aller for rad1o observations. We also thank A. Marscher, G. Chanan, and M. Urry for a critical reading of the paper. 
REFERENCES

Blanford, R.D., and Rees, M.J., 1978, In P1ttsburgh Conference on BL Lac Objects, ed. A.M. Wolfe (P1ttsburgh: University of P1ttsburgh), p. 378 .

Burb1dge, G.R., Jones, T.W., and O־De11, S.L. 1974, Ap. J., 193, 43. Cohen, M.M. et a1. 1971, Ap. J., 170, 207.

Enn18, D.J., Neugebauer, G., Werner, M., 1982, Ap. J. 262, 460.

Glaccont, R., et al. 1979, Ap. J., 230, 540.

Kellermann, K.I., et al. 1970, Ap. J., 161, 803.

Kellermann, K.I., et al. 1971, Ap. J., 169, 1.

Kr188, G. A., Canlzares, C.R., and Rlcker, G.R., 1980, Ap. J., 242, 492.

Ku, W.H.-M., Helfand, D.J., and Lucy, L.B. 1980, Nature, 288, 323.

Ledden, J.E. and O־Del1, S.L. 1983, Ap. J., 270, 434.

Kuhr, H. et a1. 1981, Astron. Astrophys. Supp1. 45, 367.

Malkan, M.A., and Sargent, W.W. 1982, Ap. J. 254, 22.

Malkan, M.A. 1983, Ap. J., 268, 582.

Madejsk1, G., and Schwartz, D.A. 1983, (submitted to Ap. J.).

Marscher, A.P., et al. 1979, Ap. J., 233, 498.

Marscher A.P. 1983, Ap. J. 264, 296.

Marscher, A.P. and Broder1ck, J.J. 1981, Ap. J. 249, 406.

Owen, F.N., Helfand, D.J., and Spangler, S.R. 1981, Ap. J. (Letters), 250, L55.

Owen, F.N., Spangler, S.R., Cotton, W.D., 1980, Astron. J., 85, 357.

Owen, F.N., Porcas, R.W., Muffson, S.L., and Moffett, T.J. 1978, A.J., 83, 685.

Perola, G.C. 1984, Physica Scripta (In Pres8). 
Preston, R.A., Morabito, D.D., W1lliams, J.G., Faulkner, J., N1colson, J.D., Jauncy, D.L., 1983, (oubmitted to A.J.).

Relchert, G.A., Mason, K.0., Thorstensen, J.R., Bowyer, S., 1982, Ap.J., 260,437 .

Schaffer, D.B. 1983, (private communication).

Scheuer, P.A.G., and Readhead, A.C.S. 1979, Nature, 277, 182.

Tananbaum, H. et. al. 1979, Ap. J.(Letter8), 234, L9.

Tananbaum, H., Wardle, J.F.C., Zamorant, G., and Avn1, Y. 1983, Ap. J. 268,60 .

Urry, C.M., and Mushotzky, R.F. 1982, Ap. J., 253, 38

Wampler, J.E., Gaskell, C.M., Burke, W.L., and Baldwin, J. A. 1984, Ap. $\mathrm{J} ., 276,403$.

W1ttels, J.J. et al. 1975, Ap. J., 196, 13.

Zamorant, G., 1983, (preprint).

Zamoran1, G. et. al. 1981 , Ap. J., $245,357$. 


\section{FIGURE CAPTIONS}

Figure 1: The histogram is for the sample reported in this paper. The average value for this sample $(\mu)$ is given with the error bar. The distribution of mean X-ray to optical spectral index is also given for active galaxies. $\alpha_{0 x}$ for Seyferts is from Kriss et a1. (1980).

Figure 2: VLBI observations are shown with the calculated angular size, assuming a spherical source.

Figure 3: Contours of $\delta$ are shown for $B$ v/c vs. $\theta$ (angle between jet and observer).

Figure 4: X-ray luminosity vs. optical luminosity and the best fit 1 ine. 
TABLE 1

OBSERVATIONS OF VARIABLE RADIO BRIGHT SOURCES

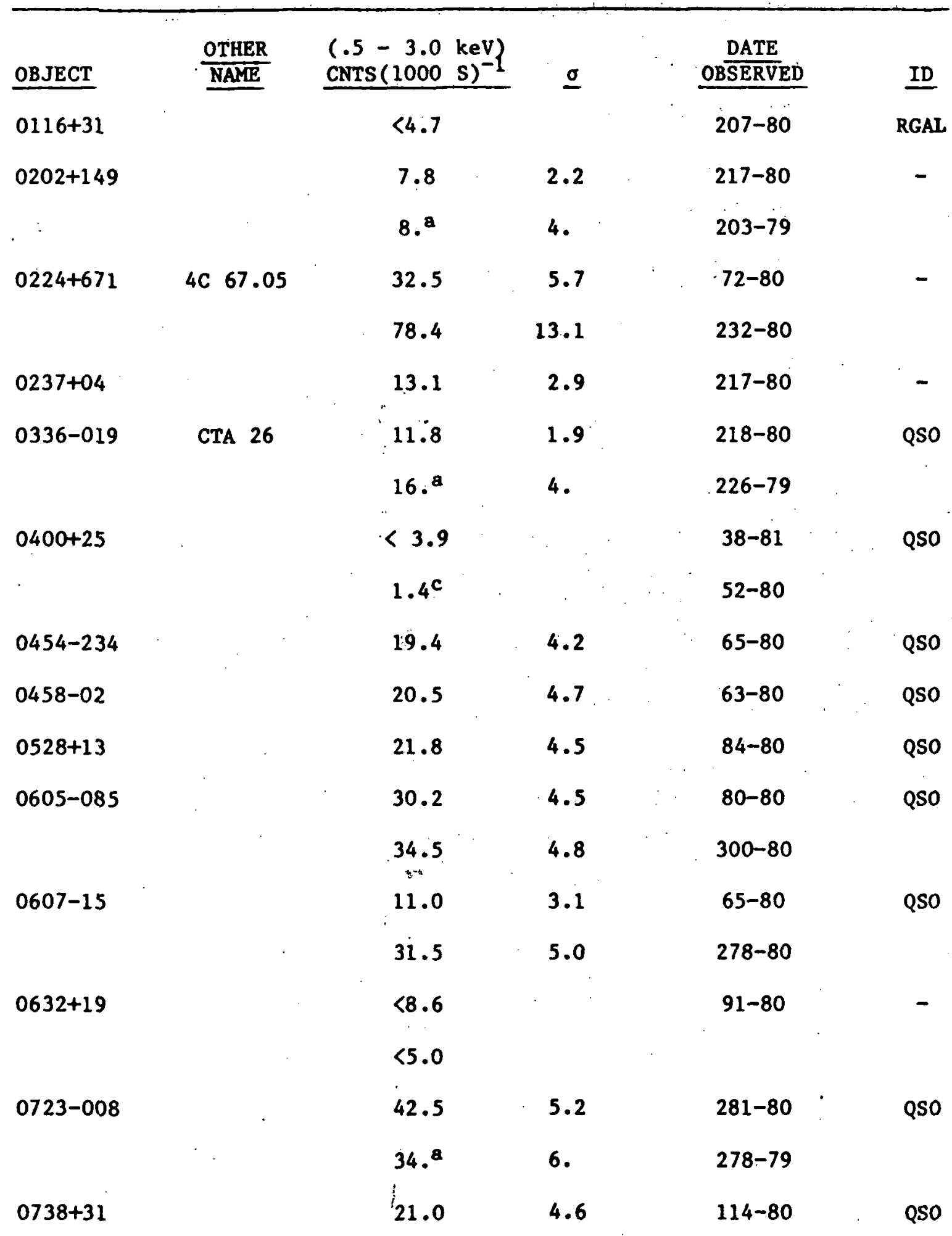




\begin{tabular}{|c|c|c|c|c|c|}
\hline & & $24.5^{c}$ & 5.0 & $299-80$ & \\
\hline \multirow[t]{2}{*}{$0834-20$} & & 19.2 & 3.0 & & \\
\hline & & 28.4 & 5.8 & $98-81$ & \\
\hline $1117+14$ & & $<15$ & & $346-80$ & QSO \\
\hline \multirow[t]{2}{*}{$1127-14$} & & 47.4 & 6.0 & $1-80$ & QSO \\
\hline & & 41.3 & 6.1 & $4-81$ & \\
\hline $1335-12$ & & $126 . \dot{4}$ & 12.7 & $196-80$ & - \\
\hline $1354-15$ & & 18.3 & 3.8 & $216-80$ & QSO \\
\hline $1358+62$ & & $<10.7$ & & $1-80$ & RGAL \\
\hline \multirow[t]{2}{*}{$1422+202$} & & $<4.0$ & & $190-80$ & QSO \\
\hline & & $18 .^{b}$ & 4. & $20-80$ & \\
\hline $1502+10$ & OR 103 & $<7.5$ & & $220-80$ & QSO \\
\hline \multirow[t]{2}{*}{$1504-167$} & & 32.6 & 6.1 & $41-80$ & QSO \\
\hline & & 21.7 & 4.7 & $211-80$ & \\
\hline $1548+05$ & & 19.8 & 4.4 & $239-80$ & Qso \\
\hline $1555+001$ & DA 393 & $<7.3$ & & $41-80$ & QSO \\
\hline \multirow[t]{2}{*}{$1611+34$} & DA 406 & 12.5 & 3.1 & $228-80$ & QSO \\
\hline & & 20.2 & 3.4 & $10-81$ & \\
\hline $1730-13$ & NRAO 530 & 29.2 & 2.8 & $76-81$ & QSO \\
\hline $1739+522$ & & 23.1 & 4.1 & $99-80$ & QSO \\
\hline $1749+096$ & oT 081 & 29.3 & 5.3 & $260-80$ & - \\
\hline $1823+56$ & & 39.5 & 5.8 & $98-80$ & QSO \\
\hline $1908-20$ & ov -213 & 64.4 & 7.8 & $97-80$ & - \\
\hline \multirow[t]{2}{*}{$2033+18$} & & $<4.9$ & & $123-80$ & - \\
\hline & & $<8.0$ & & $108-81$ & \\
\hline $2148+14$ & & 2.8 & 1.5 & $162-80$ & - \\
\hline
\end{tabular}




\begin{tabular}{rrrccr}
$2201+31$ & 4 C 31.63 & 93.0 & 7.8 & $167-80$ & QSO \\
$2230+11$ & CTA 102 & 118.3 & 22.9 & $356-80$ & QSO \\
& & $65 . \mathrm{b}$ & 5. & $328-79$ & \\
\hline
\end{tabular}

${ }^{\mathrm{a}}$ Owen et a1. (1981)

bamoran1 et al. (1981)

$c_{\text {Ledden and O'Dell (1983) }}$ 


\section{TABLE 2}

RADIO, OPTICAL, AND X-RAY PROPERTIES OF RADIO SOURCES

\begin{tabular}{|c|c|c|c|c|}
\hline OBJECTS & $\mathbf{z}$ & $\begin{array}{l}L_{x}(.5-4.5 \mathrm{kev}) \\
\times 10^{45} \mathrm{ergs}^{-1} \\
\end{array}$ & $\alpha_{\text {ox }}$ & $a_{\text {ro }}$ \\
\hline $0116+31$ & 0.059 & $<.0058$ & $>1.70$ & .48 \\
\hline $0237+04$ & .978 & $3.20 \pm .70$ & 1.30 & .68 \\
\hline $0336-019$ & .852 & $2.68 \pm .43$ & 1.29 & .84 \\
\hline \multirow[t]{2}{*}{$0400+25$} & 2.109 & $<5.70$ & $>1.71$ & .71 \\
\hline & & $<1.25$ & $>1.96$ & \\
\hline $0458-02$ & 2.286 & $15.6 \pm 3.6$ & 1.48 & .70 \\
\hline \multirow[t]{2}{*}{$0607-15$} & .324 & $.34 \pm .10$ & 1.36 & .67 \\
\hline & & $.94 \pm .15$ & 1.20 & \\
\hline $0723-00$ & .1280 & $.19 \pm .02$ & 1.00 & .70 \\
\hline \multirow[t]{2}{*}{$0738+31$} & .630 & $2.08 \pm .46$ & 1.45 & .66 \\
\hline & & $2.43 \pm .31$ & 1.42 & \\
\hline \multirow[t]{2}{*}{$1127-14$} & 1.187 & $12.73 \pm 1.65$ & 1.41 & .72 \\
\hline & & $13.74 \pm 2.06$ & 1.40 & \\
\hline $1422+20$ & .871 & $<1.42$ & $>1.42$ & .64 \\
\hline $1502+10$ & 1.833 & $<7.84$ & $>1.52$ & .55 \\
\hline \multirow[t]{2}{*}{$1504-167$} & .876 & $5.63 \pm 1.07$ & 1.18 & .83 \\
\hline & & $4.08 \pm .90$ & 1.23 & \\
\hline $1555+01$ & 1.770 & $<6.04$ & $>1.35$ & .83 \\
\hline \multirow[t]{2}{*}{$1611+34$} & 1.401 & $6.96 \pm 1.74$ & 1.45 & .75 \\
\hline & & $10.84 \pm 1.84$ & 1.31 & \\
\hline $1730-13$ & .908 & $5.60 \pm .56$ & 1.33 & .65 \\
\hline $1739+522$ & 1.375 & $14.19 \pm 2.55$ & 1.22 & .78 \\
\hline $2201+31$ & .297 & $2.62 \pm .21$ & 1.37 & .57 \\
\hline $2230+11$ & 1.037 & $41.05 \pm 1.72$ & 1.08 & .78 \\
\hline
\end{tabular}




\section{PREDICTED INVERSE COMPTON FLUX IN}

\section{5-4.5 keV BAND}

\begin{tabular}{|c|c|c|c|c|c|c|c|}
\hline OBJECT & $\mathbf{z}$ & $v_{m}\left(\mathrm{GHz}_{2}\right)$ & $\begin{array}{c}\mathbf{S}_{m} \\
\text { (JANSKYS) }\end{array}$ & $\theta(\mathrm{MAS}) *$ & $\begin{array}{c}F_{\text {PRED }} \\
110^{-13} \text { erg }\end{array}$ & $\begin{array}{c}\text { FoBs } \\
\mathrm{m}^{-2} \text { sec }^{-1}\end{array}$ & $\delta^{* *}$ \\
\hline CTA 26 & .852 & 5.0 & 2.8 & 1.4 & 80 & 3.9 & 1.8 \\
\hline DA406 & 1.401 & 0.4 & 3.3 & 1.0 & 3.9E11 & 3.2 & 160 \\
\hline NRAO 530 & 0.908 & 10.0 & 5.8 & 1.7 & 8.7 & 6.9 & 1.1 \\
\hline $4 C 31.63$ & 0.297 & 3.0 & 3.0 & 1.1 & $4.3 \mathrm{E} 3$ & 49 & 2.4 \\
\hline DA 393 & 1.770 & 10.2 & 2.4 & .92 & 69.8 & $<1.1$ & 2.3 \\
\hline $0400+25$ & 2.109 & 1.5 & 1.6 & 1.3 & $1.0 \mathrm{E} 6$ & $<.58$ & 18 \\
\hline OR 103 & 1.833 & 5.0 & 2.2 & .97 & 4.1E3 & $<1.2$ & 5.1 \\
\hline $0738+31$ & 0.630 & 2.0 & 2.3 & .80 & 1.7E6 & 6.6 & 12 \\
\hline $1739+52$ & 1.375 & 2.0 & 1.2 & 1.5 & 2.7E3 & 5.5 & 3.5 \\
\hline
\end{tabular}

*UPPER LIMIT

* LOWER LIMIT 


\section{ANGULAR SIZE OF COMPACT RADIO SOURCE}

\begin{tabular}{|c|c|c|c|c|}
\hline SOURCE & $\lambda(\mathrm{CM})$ & $\begin{array}{c}\text { BASELINE } \\
\left(10^{6} \lambda\right)\end{array}$ & VISIBILITY & $\begin{array}{l}\text { SIZE } \\
\text { (MAS) } \\
\end{array}$ \\
\hline (1) CTA26 & 3.8 & 100 & .6 & 1.4 \\
\hline (1)DA406 & 3.8 & 100 & .4 & 1.0 \\
\hline (3) NRA0530 & 6 & 55 & .5 & 1.7 \\
\hline (5) $4 \mathrm{C} 31.63$ & 2.8 & 150 & .5 & 1.1 \\
\hline (2)DA393 & 13 & 70 & .91 & .92 \\
\hline (4) $0400+25$ & 13 & 80 & .36 & 1.3 \\
\hline (6)OR103 & 3.8 & 100 & .63 & .97 \\
\hline (5) $0738+31$ & 2.8 & 150 & .3 & .80 \\
\hline (5) $1739+52$ & 3.8 & 100 & .5 & 1.5 \\
\hline $\begin{array}{l}\text { (1) COHEN et al. 1 } \\
\text { (2) KELLERMANN } \\
\text { (3) KELLERMANN }\end{array}$ & $\begin{array}{l}1) \\
\text { 1. }(1970) \\
(1971)\end{array}$ & \multicolumn{3}{|c|}{$\begin{array}{l}\text { (4) PRESTON R.A., et al. (1983) } \\
\text { (5)SCHAFFER D., (1983) } \\
\text { (6)WITTLES et al. (1975) }\end{array}$} \\
\hline
\end{tabular}




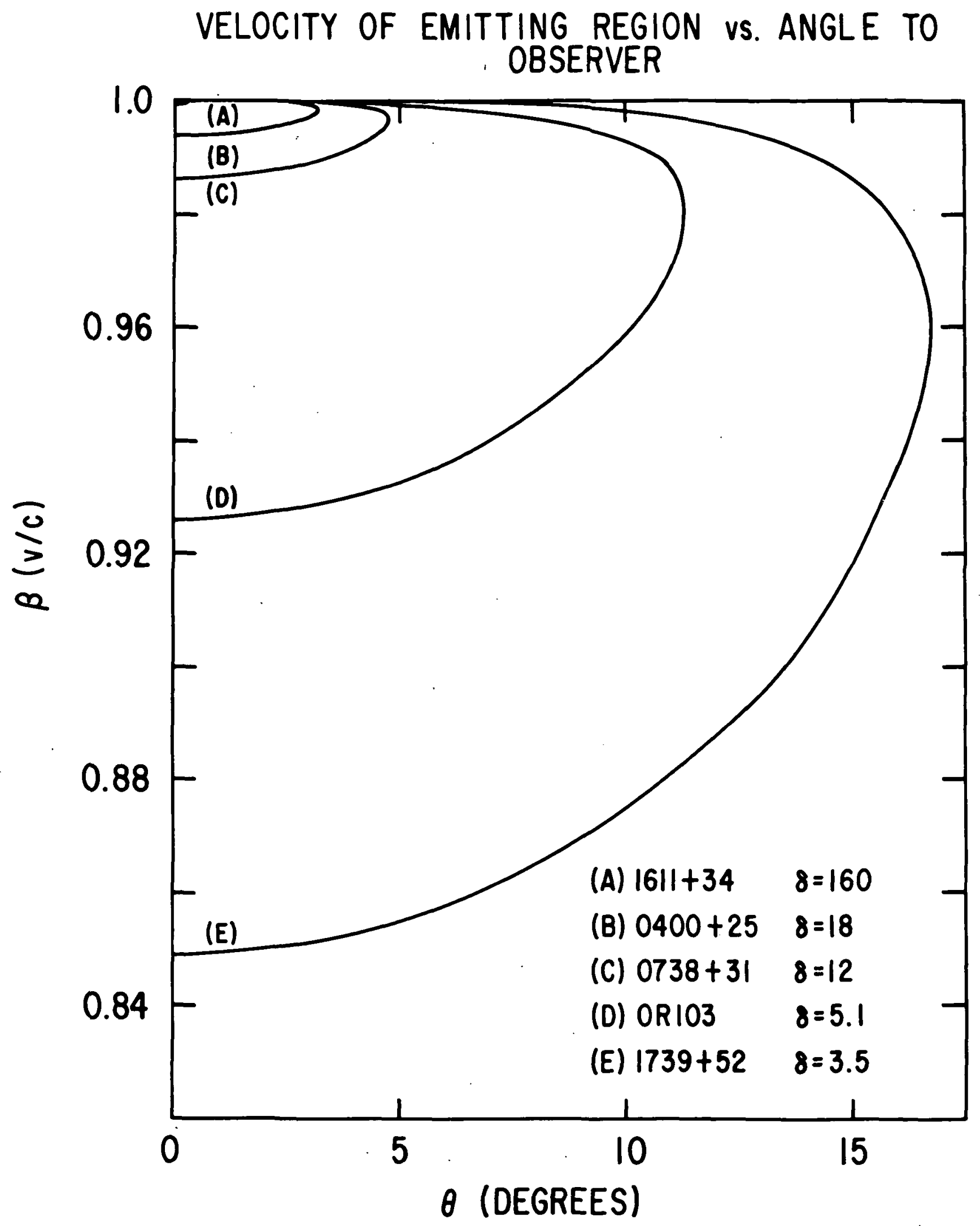




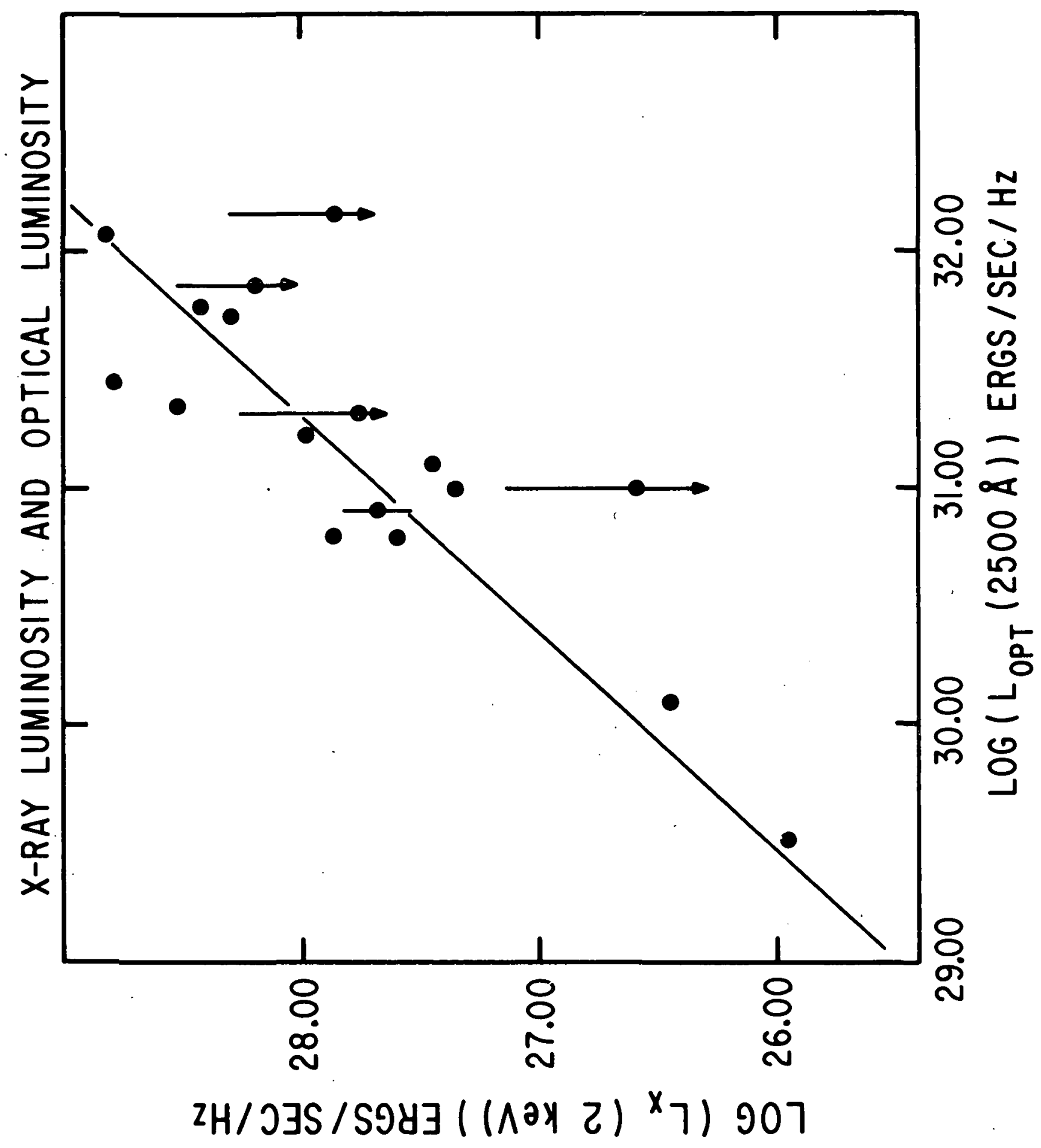




\section{BIBLIOGRAPHIC DATA SHEET}

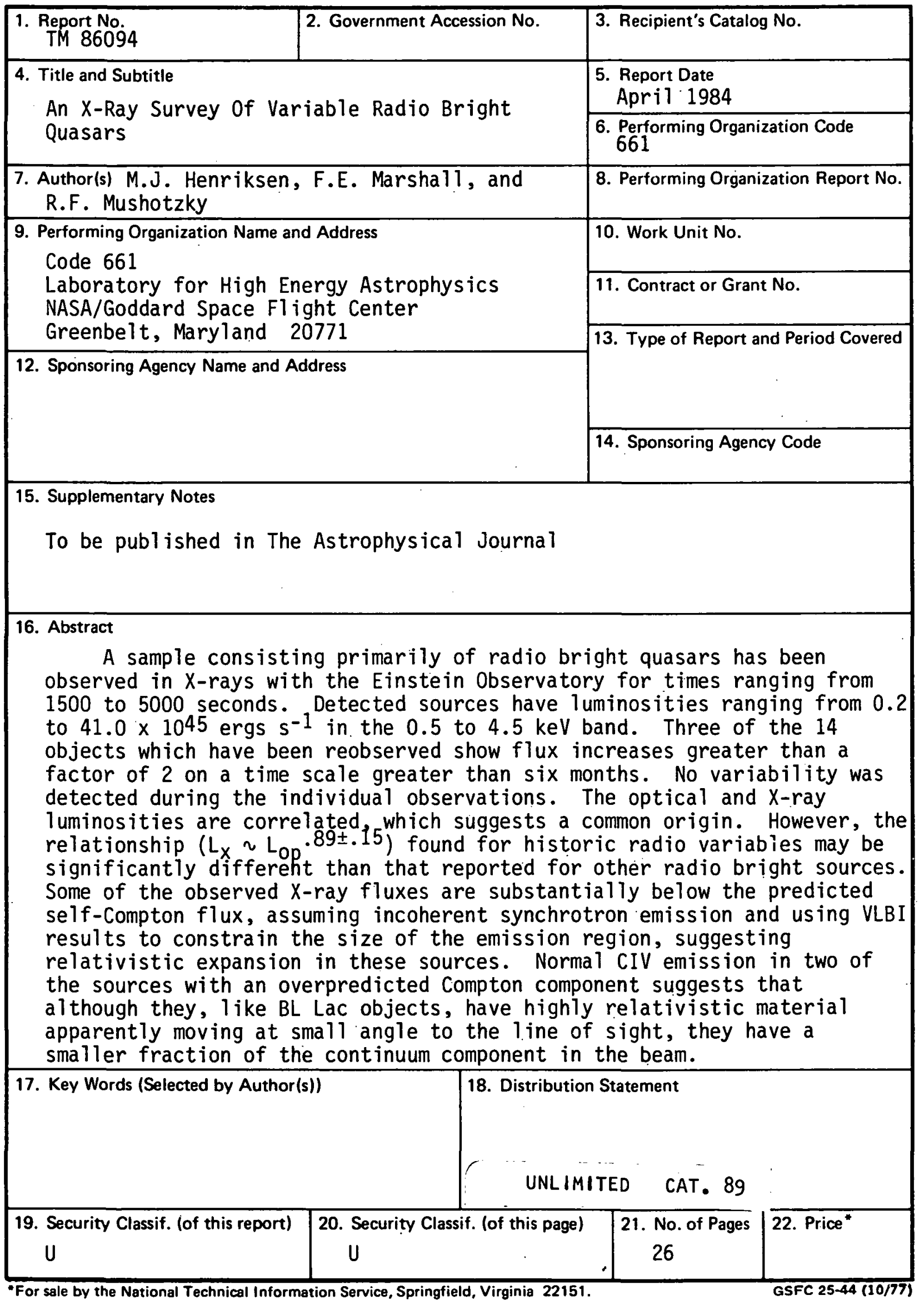

